\title{
Post-ablation lymphocytic esophagitis in Barrett esophagus with high grade dysplasia or intramucosal carcinoma
}

\author{
Juliana Kissiedu ${ }^{1}$, Prashanthi N Thota ${ }^{2}$, Tushar Gohel ${ }^{2}$, Rocio Lopez ${ }^{3}$ and Ilyssa O Gordon ${ }^{1}$ \\ ${ }^{1}$ Department of Pathology, Cleveland Clinic, Cleveland, OH, USA; ${ }^{2}$ Department of Gastroenterology, \\ Cleveland Clinic, Cleveland, OH, USA and ${ }^{3}$ Quantitative Health Science, Lerner Research Institute, \\ Cleveland Clinic, Cleveland, OH, USA
}

\begin{abstract}
In patients who have undergone ablation therapy for treatment of Barrett's esophagus with dysplasia, histologic features of eosinophilic esophagitis, but not lymphocytic esophagitis, have been described. We evaluated for histologic evidence of eosinophilic esophagitis and lymphocytic esophagitis and correlated with endoscopic findings in this population. A single-institution Barrett's esophagus registry was searched for patients who had received radiofrequency ablation, cryotherapy, or both for treatment of Barrett's esophagus with dysplasia. Clinical and endoscopic data were collected and biopsies were reviewed for inflammation and reactive changes at three time points: pre-intervention, first surveillance after ablation therapy, and most recent surveillance. Of the 173 patients initially identified, 102 met the inclusion criteria. Intraepithelial eosinophils were increased at first surveillance $(60 \%, P=0.096)$ and last surveillance $(69 \%, P=0.048)$ compared with pre-intervention $(50 \%)$, although histologic evidence of post-ablation eosinophilic esophagitis was not significant. Prevalence of lymphocytic esophagitis was significantly higher at first surveillance $(17 \%, P=0.02)$ and at last surveillance (43\%, $P<0.001$ ), compared with pre-intervention (7\%). Smoking, hyperlipidemia, and cryotherapy were identified as independent risk factors for developing histologic lymphocytic esophagitis. This is the first report that histologic evidence of lymphocytic esophagitis increased over time in patients undergoing ablation for Barrett's esophagus with dysplasia. Though the pathophysiology of lymphocytic esophagitis remains unknown, patients in our study with a history of smoking, hyperlipidemia, or cryotherapy were more likely to develop post-ablation lymphocytic esophagitis.
\end{abstract}

Modern Pathology (2016) 29, 599-606; doi:10.1038/modpathol.2016.50; published online 11 March 2016

Endoscopic ablation is commonly performed for the treatment of Barrett's esophagus with high grade dysplasia and residual Barrett's esophagus after resection of intramucosal carcinomas. After ablation treatments, with maximal acid suppression, the ablated Barrett's esophagus is replaced with the neosquamous epithelium which is similar to the normal squamous mucosa. Neosquamous epithelium often shows regenerative changes, such as basal cell hyperplasia and elongation of the lamina propria papillae. These features are usually considered to be indicative of epithelial repair when seen in the early post-ablation period. ${ }^{1}$

Correspondence: Dr IO Gordon, MD, PhD, Department of Pathology, Cleveland Clinic, 9500 Euclid Avenue, L25, Cleveland, $\mathrm{OH} 44195$, USA.

E-mail: gordoni@ccf.org

Received 11 November 2015; revised 5 February 2016; accepted 8 February 2016; published online 11 March 2016
Esophageal eosinophilia has been recently identified in biopsies from patients who have undergone radiofrequency ablation for the treatment of Barrett's esophagus with low grade dysplasia, high grade dysplasia, or intramucosal carcinoma. Owens et $a l^{2}$ described eosinophilic esophagitis in patients who had undergone photodynamic therapy or radiofrequency ablation with or without previous endoscopic mucosal resection. Halsey et $a l^{3}$ described esophageal eosinophilia in patients who had undergone radiofrequency ablation or cryotherapy for treatment of Barrett's esophagus-related changes. The long-term clinical consequences of this post-ablation esophageal eosinophilia and whether it is related to eosinophilic esophagitis is not known. In addition, there is no study describing histologic evidence of other forms of esophagitis like the newly described entity, lymphocytic esophagitis, in patients who have undergone ablation therapy. 
Table 1 Demographic and baseline endoscopic characteristics

\begin{tabular}{lcc}
\hline Factor & $\mathrm{n}$ & Summary \\
\hline Age (years) & 102 & $67 \pm 10$ \\
Gender & 102 & \\
$\quad$ Male & 92 & \\
Ethnicity & & $88(93)$ \\
$\quad$ Caucasian & & $2(2)$ \\
African American & & $2(2)$ \\
$\quad$ Others & 101 & \\
Smoking & & $46(46)$ \\
Non-smoker & & $44(44)$ \\
Ex-smoker & 100 & $11(11)$ \\
$\quad$ Current smoker & & $53(53)$ \\
Alcohol use & & $37(37)$ \\
Never & & $7(7)$ \\
Mild & & $3(3)$ \\
Moderate & 89 & $31 \pm 7$ \\
Ex-alcohol use & & \\
Body mass index & & \\
& 102 & $9(9)$ \\
Medications (non-exclusive) & 102 & $48(47)$ \\
$\quad$ Nonsteroidal anti-inflammatory & 102 & $55(54)$ \\
Aspirin & 102 & $72(71)$ \\
Statins & 102 & $101(99)$ \\
Anti-hypertensives & 102 & $2(2)$ \\
Proton pump inhibitors & 102 & $61(60)$ \\
Histamine H2-receptor antagonists & 102 & $22(22)$ \\
Hypertension & 102 & $54(53)$ \\
Diabetes & 102 & $5 \pm 3$ \\
Hyperlipidemia & 101 & $3 \pm 2$ \\
Length of Barrett's esophagus (cm) & & \\
Length of hiatal hernia (cm) & & \\
& & \\
\end{tabular}

Values presented as mean \pm standard deviation, median (P25, P75) or $N$ (column \%)

Lymphocytic esophagitis is an emerging clinicopathologic entity that was originally described by Rubio et al. ${ }^{4}$ Lymphocytic esophagitis is characterized by an increased number of intraepithelial lymphocytes in the peripapillary region with associated spongiosis and occasionally rare granulocytes. ${ }^{4}$ The pathophysiology of this entity remains unclear and it is considered a diagnosis of exclusion. ${ }^{5,6}$

Our study aims were to (i) evaluate histological changes in esophageal post-ablation squamous mucosa compared with pre-ablation, (ii) evaluate whether there is histologic evidence of the recently described entity lymphocytic esophagitis post-ablation, (iii) evaluate whether there is histologic evidence of eosinophilic esophagitis post-ablation, and (iv) evaluate whether there are any endoscopic or clinical findings associated with the histologic findings in our patient population.

\section{Materials and methods}

\section{Study Population}

All patients 18 years or older who underwent endoscopic eradication therapy for Barrett's esophagus with high grade dysplasia or intramucosal carcinoma between January 2006 and December 2011 at our institution were included in the study. Exclusion criteria were patients less than 18 years of age, patients whose biopsies were not available for histologic review, patients who had no index biopsy on file, and patients who underwent endoscopic mucosal resection only without biopsies. The study was approved by the Institutional Review Board of Cleveland Clinic, Cleveland, OH, USA.

The electronic medical record was reviewed for demographic data such as age, gender, race, comorbidities such as smoking, diabetes, hypertension, hyperlipidemia, and medication use including aspirin, non-steroidal anti-inflammatory drugs, proton pump inhibitors, and histamine H2-receptor antagonists. Endoscopic data such as presence of any visible changes including strictures, erosion, rings, and furrowing were recorded.

\section{Histologic Evaluation}

Biopsies were reviewed for inflammation and reactive changes of the squamous mucosa on formalinfixed paraffin embedded tissue cut 5-microns thick and stained with hematoxylin and eosin. Three time points were studied: pre-intervention, first surveillance, and last surveillance. Pre-intervention biopsy was the biopsy before the first radiofrequency ablation or cryoablation. First surveillance biopsy was defined as the first biopsy after ablation therapy with no endoscopic evidence of intestinal metaplasia. The last surveillance biopsy was the most recent surveillance biopsy on file or the last biopsy with no evidence of intestinal metaplasia after ablation therapy. The following criteria were used to make the diagnosis of lymphocytic esophagitis, similar to the criteria described by Cohen et al: ${ }^{5}$ (i) $\geq 20$ peripapillary lymphocytes per high power field, (ii) peripapillary lymphocytosis with spongiosis, and (iii) absence of significant neutrophils or eosinophils. Eosinophilic esophagitis was defined as greater than 15 intraepithelial eosinophils per high power field with eosinophil microabscesses. Reactive changes within the squamous epithelium that have been associated with inflammation and gastroesophageal reflux disease, specifically elongation of papillae and basal cell hyperplasia, ${ }^{7}$ were also evaluated.

\section{Endoscopic Therapy}

Patients presenting for endoscopic therapy for Barrett's esophagus had detailed endoscopic examination with white light and narrow band imaging. Endoscopic ultrasound was performed at the proceduralists' discretion. Any visible lesions such as nodules, plaques, or ulcers were resected by endoscopic mucosal resection. If at that point patients were deemed to be candidates for endoscopic therapy, they underwent radiofrequency 
Table 2 Histologic and endoscopic characteristics

\begin{tabular}{|c|c|c|c|c|c|}
\hline \multirow{2}{*}{ Factor } & \multirow{2}{*}{$\frac{\begin{array}{c}\text { Pre-intervention } \\
(\mathrm{N}=102)\end{array}}{\text { Summary }}$} & \multicolumn{2}{|c|}{$\begin{array}{l}\text { First surveillance } \\
\qquad(\mathrm{N}=102)\end{array}$} & \multicolumn{2}{|c|}{$\begin{array}{l}\text { Last surveillance } \\
\qquad(\mathrm{N}=70)\end{array}$} \\
\hline & & Summary & P-value* & Summary & P-value ${ }^{\mathrm{a}}$ \\
\hline Squamous mucosa present & $102(100)$ & $101(99)$ & 0.32 & $70(100)$ & - \\
\hline Any inflammation & $102(100)$ & $99(98)$ & 0.16 & $70(100)$ & - \\
\hline Eosinophils & $51(50)$ & $61(60)$ & 0.096 & $48(69)$ & 0.048 \\
\hline \multicolumn{6}{|l|}{ If eosinophils present: } \\
\hline Maximum eosinophils greater than 15 per high power field & $9(18)$ & $9(15)$ & 0.32 & 7 (15) & 0.41 \\
\hline Eosinophil microabscess & $5(10)$ & $6(10)$ & 0.99 & $3(6)$ & 0.99 \\
\hline Eosinophil degranulation & $15(29)$ & $24(39)$ & 0.02 & $22(46)$ & 0.16 \\
\hline Neutrophils & $31(30)$ & $44(44)$ & 0.047 & $19(27)$ & 0.55 \\
\hline Lymphocytes & $101(99)$ & $99(98)$ & 0.56 & $70(100)$ & 0.32 \\
\hline \multicolumn{6}{|l|}{ If lymphocytes present: } \\
\hline Lymphocytic esophagitis ${ }^{b}$ & $7(7)$ & $17(17)$ & 0.02 & $30(43)$ & $<0.001$ \\
\hline \multicolumn{6}{|l|}{ If squamous mucosa present: } \\
\hline Elongation of papillae & $20(20)$ & $14(14)$ & 0.26 & $13(19)$ & 0.83 \\
\hline Any spongiosis & $89(87)$ & $91(90)$ & 0.47 & $67(96)$ & 0.21 \\
\hline Basal layer hyperplasia & $42(41)$ & $52(52)$ & 0.1 & $29(41)$ & 0.99 \\
\hline \multicolumn{6}{|l|}{ If inflammation, esophagus appearance: } \\
\hline Normal & $91(93)$ & $79(82)$ & 0.004 & $58(84)$ & 0.058 \\
\hline Stricture & $3(3)$ & $7(7)$ & 0.046 & 8 (12) & 0.014 \\
\hline Multiple rings & $1(1)$ & $0(0)$ & 0.32 & $0(0)$ & 0.32 \\
\hline Longitudinal furrows & $0(0)$ & $2(2)$ & 0.16 & $0(0)$ & - \\
\hline
\end{tabular}

${ }^{\text {a }}$-values for comparison of each follow-up to pre-intervention values and include subjects who have each post-intervention available. Bowker's Test of Symmetry for eosinophil distribution and Barrett's esophagus histology, McNemar's test was used for all other categorical variables and Wilcoxon signed rank test was used for number of eosinophils. ${ }^{b}$ Lymphocytic esophagitis defined as (i) $\geq 20$ peripapillary lymphocytes per high power field, (ii) peripapillary lymphocytosis with spongiosis, and (iii) absence of significant neutrophils or eosinophils.

Values presented as mean \pm standard deviation, median $(\mathrm{P} 25, \mathrm{P} 75)$ or $N$ (column \%).

ablation or cryotherapy. Radiofrequency ablation was performed by Halo-360 and/or Halo-90 depending on the length of the Barrett's esophagus segment. Halo-360 is used for circumferential 360 degree 3-cm-long superficial ablation and is typically used for long segment Barrett's. Halo-90 is a focal ablation device mounted on endoscope for ablation of short segment Barrett's or residual Barrett's after a Halo-360 application. This application of controlled thermal energy causes water vaporization, coagulation of proteins, and cell necrosis. ${ }^{8}$ The desiccated tissue has much higher resistance to current than normal tissue. This leads to an ablation depth that is well controlled and provides a superficial depth of injury.

Cryotherapy was performed by delivering liquid nitrogen $\left(-196^{\circ} \mathrm{C}\right)$ through a long insulated catheter passed through the endoscope. This leads to rapid freezing of the tissue followed by slow thawing. This contributes to cell death through several mechanisms. ${ }^{9}$ Ablation sessions are repeated every 2-3 months until there is complete absence of visible Barrett's esophagus, at which point patients entered into the surveillance program. During surveillance, four quadrant biopsies are carried out at $1-\mathrm{cm}$ intervals from the original Barrett's esophagus segment and any visible abnormalities are biopsied, resected, or ablated at the endoscopists' discretion.

\section{Statistical Analysis}

Clinical and endoscopic findings were correlated with histologic findings for all time points. Data are presented as mean \pm standard deviation, median $\left(25^{\text {th }}, 75^{\text {th }}\right.$ percentiles) or $N(\%)$. Paired analysis was performed to assess differences in histologic findings; pre-intervention findings were compared with first and last surveillance findings separately. McNemar's tests were used for binary categorical variables, Bowker's test for symmetry was used for eosinophil distribution and Barrett's esophagus histology, and Wilcoxon signed rank test was used for number of eosinophils present. In addition, a univariable analysis was carried out to assess the factors associated with post-ablation lymphocytic esophagitis in subjects without lymphocytic esophagitis before ablation. Analysis of variance was used for continuous variables and Pearson's chi-square tests or Fisher's Exact tests were used for categorical factors. Multivariable logistic regression was also performed to assess adjusted associations with occurrence of post-ablation lymphocytic esophagitis; all variables with $P<0.10$ on the univariable analysis were considered for inclusion in the model and those with adjusted- $P<0.05$ were kept in the final model. A $P<0.05$ was considered statistically significant. SAS (version 9.4, The SAS 

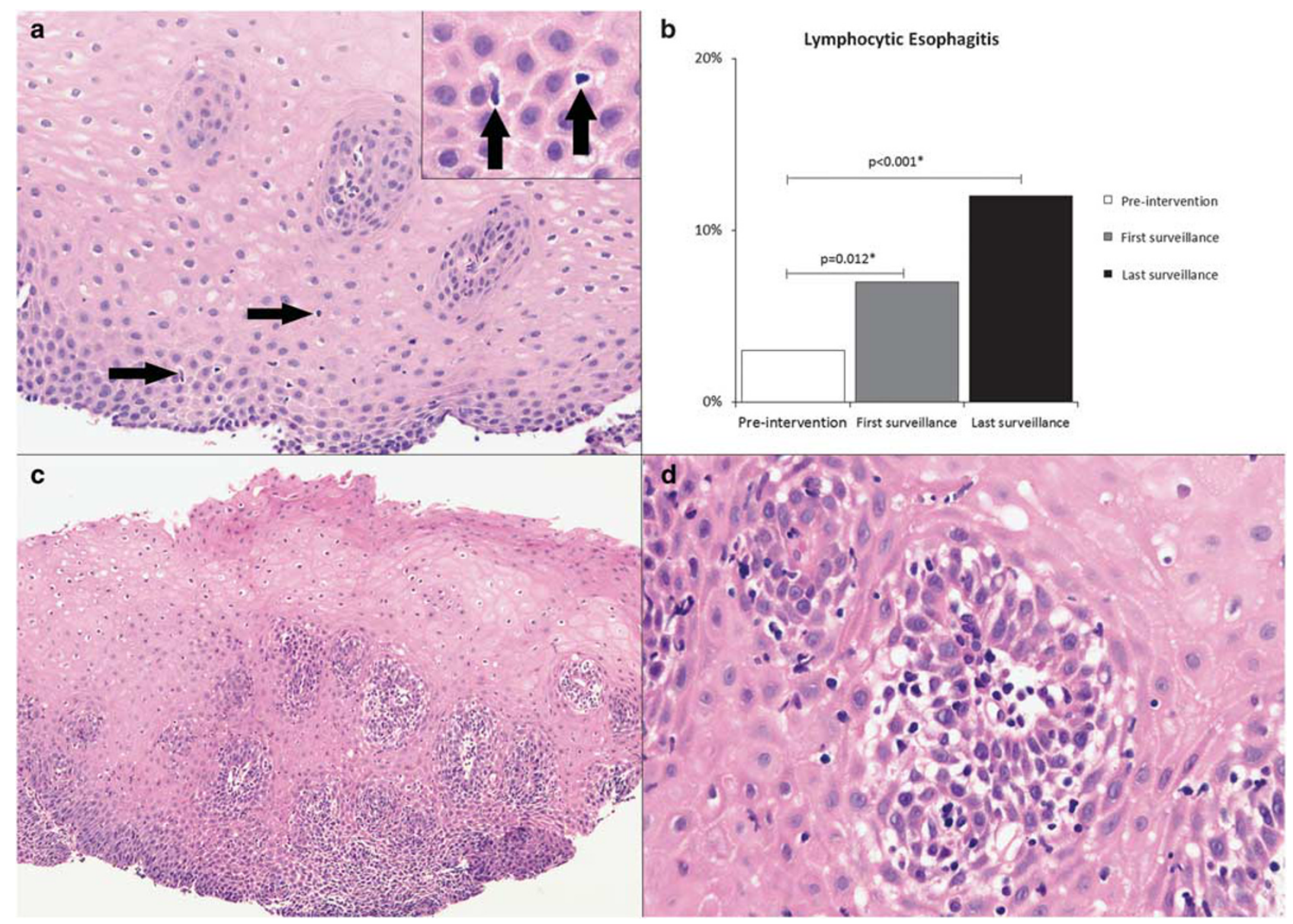

Figure 1 (a) Normal esophageal squamous mucosa has scattered intraepithelial lymphocytes, as indicated by black arrows (hematoxylin and eosin stain, original magnification, $200 \times$ and, inset, $400 \times$ ). (b) Prevalence of lymphocytic esophagitis increased in the first and last surveillance biopsies as compared with the pre-ablation biopsy. Example of post-ablation lymphocytic esophagitis characterized by increased intraepithelial peripapillary lymphocytes with associated spongiosis (intercellular edema) of the squamous epithelium, low power (c) and high power (d) (hematoxylin and eosin stain, original magnification, $100 \times$ and $400 \times$ respectively).

Institute, Cary, NC, USA) and R (version 3.0.3, The $\mathrm{R}$ Foundation for Statistical Computing, Vienna, Austria) were used to perform all analyses.

\section{Results}

\section{Patient Characteristics}

A total of 173 patients underwent endoscopic therapy for Barrett's esophagus with high grade dysplasia or intramucosal carcinoma during the study period. Patients were not included in the study because either index or surveillance biopsy slides could not be obtained for review $(n=61)$ or patients had endoscopic mucosal resection only but not ablation $(n=10)$, resulting in 102 patients included in the final analysis. Table 1 shows a summary of demographic, baseline endoscopic, and clinical characteristics.

The average time between pre-intervention biopsy and first surveillance biopsy was 280 days, and the average time between first surveillance biopsy and last surveillance biopsy was 978 days.

\section{Histology}

Intraepithelial eosinophils were identified in $50 \%$ of patients at the time of pre-intervention biopsy and this increased to $60 \% \quad(P=0.096)$ at the first surveillance and $69 \%(P=0.048)$ at the last surveillance. However, there were no significant differences between the maximum number of eosinophils per high power field or histologic evidence of eosinophilic esophagitis ( $>15$ eosinophils per high power field and eosinophil microabscess). Eosinophil degranulation and the presence of intraepi thelial neutrophils were significantly increased at the first surveillance as compared with the preintervention biopsy (39\% vs $29 \%, P=0.02$ and $44 \%$ vs $30 \%, P=0.047$, respectively), but not at the last surveillance. Esophageal erosions were also more common at the time of first surveillance as compared 


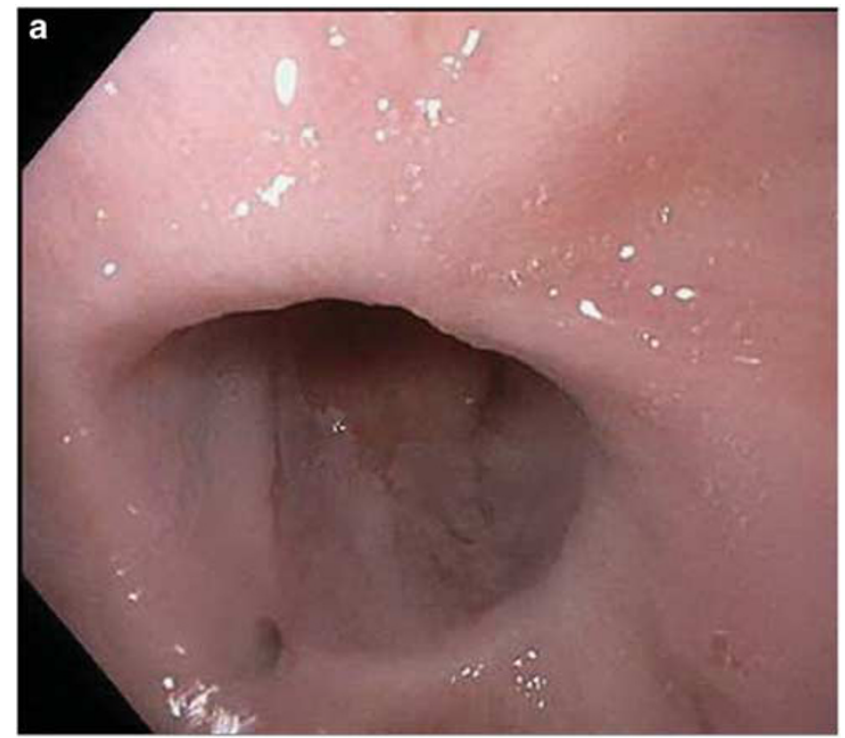

Strictures

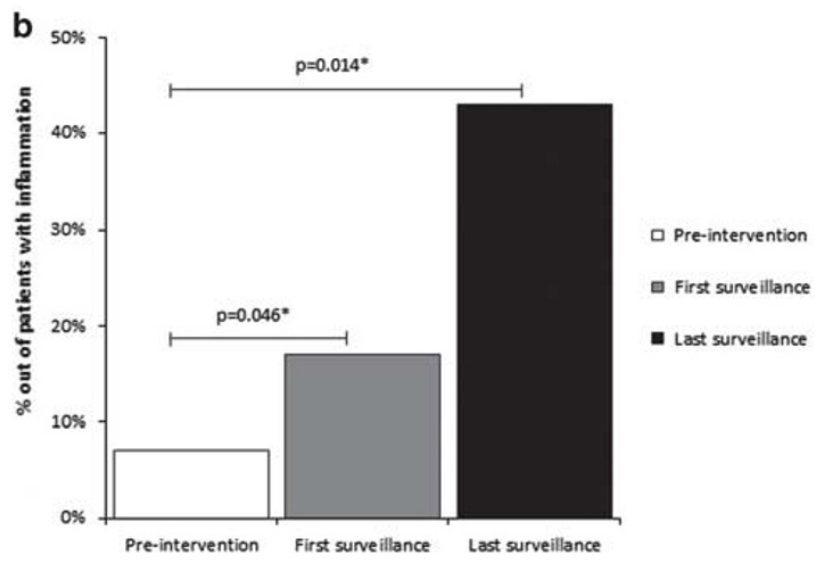

Figure 2 (a) Example of a stricture identified at endoscopy in a post-ablation patient. (b) Prevalence of endoscopically identified strictures increased in the first and last surveillance endoscopy as compared with the pre-ablation endoscopy.

with pre-intervention (12 vs $4 \%, P=0.035$ ), but not at the last surveillance. Table 2 shows detailed histologic findings.

Nearly all patients had intraepithelial lymphocytes at each biopsy (Table 2 and Figure 1a). Prevalence of peripapillary lymphocytes with spongiosis in the absence of significant neutrophils or eosinophils (defining lymphocytic esophagitis) was significantly higher at the first surveillance $(17 \%, P=0.02)$ and at the last surveillance $(43 \%$, $P<0.001)$, as compared with pre-intervention $(7 \%)$ (Figure 1b-d). Similarly, in patients with any type of inflammation, prevalence of endoscopically identified strictures (Figure 2) was significantly higher during both the first surveillance $(7 \%, P=0.046)$ and last surveillance $(12 \%, P=0.014)$, as compared with pre-intervention $(3 \%)$. Strictures and erosions were not specifically associated with post-ablation lymphocytic esophagitis.

\section{Clinical Correlation}

Patients with post-ablation lymphocytic esophagitis were more likely to have a history of smoking $(P=0.03)$ and have received cryotherapy $(P=0.015)$ (Table 3). In addition, there was a trend toward association with use of statins and hyperlipidemia. These four factors were further explored in multivariable analysis. The final logistic regression model contained smoking, hyperlipidemia, and cryotherapy (Table 4). After adjusting for hyperlipidemia and cryotherapy, patients who had a history of smoking were three times more likely to develop post-ablation lymphocytic esophagitis $(P=0.038)$. In addition, patients with hyperlipidemia were 3.5 times more likely to have post-ablation lymphocytic esophagitis than those without hyperlipidemia $(P=0.017)$. Lastly, after adjusting for smoking and hyperlipidemia, patients who received cryotherapy were almost five times more likely to develop post-ablation lymphocytic esophagitis $(P=0.005)$.

\section{Discussion}

We found that histologic evidence of lymphocytic esophagitis increased over time in patients undergoing ablation for Barrett's esophagus with dysplasia or intramucosal carcinoma. This has not been previously described. Patients with a history of smoking or hyperlipidemia and patients who had undergone cryotherapy were more likely to develop post-ablation lymphocytic esophagitis. The pathophysiology of lymphocytic esophagitis has not been clearly defined. Lymphocytic esophagitis has been described as a clinicopathologic condition ${ }^{4}$ and in a series of 42 lymphocytic esophagitis cases, no association was found between histologic lymphocytic esophagitis and any clinical characteristic, medication, concurrent disease, or symptoms, suggesting that the finding may be a response to pathologic stimuli. ${ }^{10}$ Our study supports this suggestion, and further suggests that in patients who undergo endoscopic procedures like ablation and biopsy, histologic lymphocytic esophagitis could be a non-specific response to esophageal mucosal injury, and that a history of hyperlipidemia, smoking, or cryotherapy may be related to an increased susceptibility to this type of immune response. This phenomenon may be similar to the reported association of erosive esophagitis in patients with metabolic syndrome because of an increase in proinflammatory adipokines. ${ }^{11}$ Hyperlipidemia is one of the components of metabolic syndrome and, though not extensively studied, may have a similar role related to a proinflammatory qstate in our patients who developed post-ablation lymphocytic esophagitis.

Lymphocytic esophagitis is an emerging entity which is being diagnosed with increasing frequency. ${ }^{5}$ The clinical presentation may be similar 
Table 3 Post-ablation lymphocytic esophagitis

\begin{tabular}{|c|c|c|c|}
\hline & $\begin{array}{c}\text { No post-ablation lymphocytic } \\
\text { esophagitis }(\mathrm{N}=62)\end{array}$ & $\begin{array}{c}\text { Post-ablation lymphocytic } \\
\text { esophagitis }(\mathrm{N}=33)\end{array}$ & \\
\hline Factor & Summary & Summary & $\mathrm{P}$-value \\
\hline Age (years) & $68 \pm 11$ & $67 \pm 10$ & 0.61 \\
\hline Gender & & & 0.96 \\
\hline Female & $11(18)$ & $6(18)$ & \\
\hline Male & $51(82)$ & $27(82)$ & \\
\hline Caucasian & $56(98)$ & $28(90)$ & $0.12^{\mathrm{F}}$ \\
\hline Any smoking & $30(48)$ & $23(72)$ & 0.03 \\
\hline Any alcohol & $28(46)$ & $17(53)$ & 0.51 \\
\hline Body mass index & $31 \pm 7$ & $31 \pm 7$ & 0.96 \\
\hline \multicolumn{4}{|l|}{ Medications } \\
\hline Aspirin & $30(48)$ & $16(49)$ & 0.99 \\
\hline Nonsteroidal anti-inflammatory & $4(7)$ & $5(15)$ & 0.17 \\
\hline Statins & $31(50)$ & $23(70)$ & 0.065 \\
\hline Antihypertensive & $42(68)$ & $26(79)$ & 0.26 \\
\hline Proton pump inhibitors & $62(100)$ & $32(97)$ & $0.35^{\mathrm{F}}$ \\
\hline Histamine H2-receptor antagonists & $2(3)$ & $0(0)$ & $0.54^{\mathrm{F}}$ \\
\hline Family history of esophageal cancer & $1(2)$ & $3(9)$ & $0.13^{\mathrm{F}}$ \\
\hline \multicolumn{4}{|l|}{ Comorbidities (non-exclusive) } \\
\hline Hypertension & $36(58)$ & $21(64)$ & 0.6 \\
\hline Diabetes & $12(19)$ & $8(24)$ & 0.58 \\
\hline Hyperlipidemia & $30(48)$ & $22(67)$ & 0.088 \\
\hline Fundoplication & $2(3)$ & $1(3)$ & $0.99^{\mathrm{F}}$ \\
\hline Length of Barrett's esophagus (cm) & $6 \pm 3$ & $5 \pm 4$ & 0.69 \\
\hline Length of hiatal hernia (cm) & $3 \pm 2$ & $3 \pm 1$ & 0.37 \\
\hline Any endoscopic mucosal resection & $34(55)$ & $21(64)$ & 0.41 \\
\hline Any radiofrequency ablation & $39(63)$ & $17(52)$ & 0.28 \\
\hline Any cryotherapy & $29(47)$ & $24(73)$ & 0.015 \\
\hline
\end{tabular}

Values presented as mean \pm standard deviation with analysis of variance or $N$ (column \%) with Fisher's Exact test $\left({ }^{\mathrm{F}}\right.$ ) or Pearson's chi-square test.

Table 4 Post-ablation lymphocytic esophagitis: multivariable logistic regression

\begin{tabular}{lcc}
\hline Factor & $\begin{array}{c}\text { Odds ratio } \\
\text { (95\% confidence interval) }\end{array}$ & P-value \\
\hline Any smoking & $2.9(1.06,7.9)$ & 0.038 \\
Hyperlipidemia & $3.5(1.2,9.7)$ & 0.017 \\
Any cryotherapy & $4.6(1.6,13.1)$ & 0.005 \\
\hline
\end{tabular}

to that of eosinophilic esophagitis or gastroesophageal reflux disease, with dysphagia, chest pain, heartburn, and odynophagia. ${ }^{5,6}$ Symptom improvement has been reported with proton pump inhibitors, ${ }^{5}$ bringing into question whether this entity is actually a form of gastroesophageal reflux disease, although others have postulated that it may be a common histologic response to a variety of possible etiologic pathways. ${ }^{10} \mathrm{~A}$ range of endoscopic findings in patients with lymphocytic esophagitis have been reported, from normal to rings, strictures, or other features usually associated with eosinophilic esophagitis. ${ }^{5,6}$ In our study, esophageal strictures and erosions were found.

We did not find eosinophilic esophagitis in patients who have undergone ablation therapy for the treatment of Barrett's esophagus. This differs from a previous report which did find eosinophilic esophagitis in the setting of ablation therapy for Barrett's esophagus with dysplasia. ${ }^{2}$ We did find an increased number of patients with intraepithelial eosinophils in the first surveillance biopsy, which, along with increased neutrophils and endoscopic erosions/fissures at the same time point, may be attributable to an injury/repair process related to the ablation procedure or to underlying gastroesophageal reflux disease. This finding is similar to that of Halsey et al, who described esophageal eosinophilia ( $\geq 5$ eosinophils per high power field), but not specifically eosinophilic esophagitis, in Barrett's esophagus patients post ablation. ${ }^{3}$

Although the current definition of lymphocytic esophagitis includes having no more than rare granulocytes, it is known that lymphocytes are present in both gastroesophageal reflux disease and eosinophilic esophagitis, ${ }^{12}$ and it has been postulated that the phenotype of intraepithelial lymphocytes may have a role in the development of Barrett's esophagus. ${ }^{13}$ The role of lymphocytes in relation to neutrophils and eosinophils has also been studied in the context of infectious esophagitis. ${ }^{14}$

A few lymphocytes are normally present in the esophageal squamous mucosa, however, a normal number is not defined. An early study by 
Mangano et $a l^{15}$ reported 0.8 mononuclear cells per high power field in benign squamous epithelium in areas away from resected tumor, and up to 6.3 mononuclear cells per high power field in cases with active esophagitis and infection. A type of intraepithelial $\mathrm{T}$ lymphocyte, which has irregular nuclear contours, or squiggle cells, has been reported in normal esophageal squamous epithelium, active esophagitis, infection, Barrett's esophagus, and esophageal carcinoma ${ }^{15}$ as well as in pediatric gastroesophageal reflux disease. ${ }^{16}$ Intraepithelial lymphocytes in the esophagus have also been reported in association with inflammatory bowel disease, ${ }^{17,18}$ although an association specifically between lymphocytic esophagitis and inflammatory bowel disease has not been a universal finding. ${ }^{4-6,10}$ Rarely, primarily cutaneous diseases, such as lichen planus, can present in the esophagus with a marked increase in intraepithelial lymphocytes; pill-esophagitis and medication injury to the esophagus can also have intraepithelial lymphocytosis. ${ }^{19}$

There are a few limitations to our study. All of the patients in our study had an established diagnosis of gastroesophageal reflux disease. As intraepithelial lymphocytes and eosinophils, as well as reactive changes such as spongiosis, are known to be present in gastroesophageal reflux disease, this may have had an impact on our results. We attempted to reduce this impact by counting intraepithelial eosinophils and by using definitions of eosinophilic esophagitis and lymphocytic esophagitis which would be more specific than what might be explained by gastroesophageal reflux disease alone. However, the possibility still remains, as suggested by others, that the histologic finding of lymphocytic esophagitis is within the spectrum of gastroesophageal reflux disease clinically.

Another limitation to our study is that we did not separate our patients into more specific therapy groups. Some of our patients received both radiofrequency ablation and cryotherapy so the histologic findings of lymphocytic esophagitis could be associated with the combination of radiofrequency ablation and cryotherapy and not just cryotherapy alone, although cryotherapy was an independent risk factor for lymphocytic esophagitis. In addition, because there are no specific symptoms to differentiate gastroesophageal reflux disease from lymphocytic esophagitis, we did not evaluate for symptoms in our patient population.

We describe for the first time histologic lymphocytic esophagitis in Barrett's esophagus patients who have undergone ablation therapy. In these patients, lymphocytic esophagitis was associated with prior cryoablation, hyperlipidemia, and history of smoking. We also found more patients with intraepithelial eosinophils post ablation, but no increase in eosinophilic esophagitis. Lymphocytic esophagitis is a new entity that needs further characterization as to etiology and clinical significance. It is important for a pathologist to report the histologic findings in order to help clinicians look for signs or symptoms in the patient, and exclude other histologically similar entities.

\section{Disclosure/conflict of interest}

The authors declare no conflict of interest.

\section{References}

1 Odze RD, Lauwers GY. Histopathology of Barrett's esophagus after ablation and endoscopic mucosal resection therapy. Endoscopy 2008;40:1008-1015.

2 Owens VL, Katzka DA, Lutzke LS et al. Endoscopic ablative therapy for Barrett's esophagus: a potential cause of eosinophilic esophagitis. Dis Esophagus 2012;25:33-39.

3 Halsey KD, Arora M, Bulsiewicz WJ et al. Eosinophilic infiltration of the esophagus following endoscopic ablation of Barrett's neoplasia. Dis Esophagus 2013;26: 113-116.

4 Rubio CA, Sjodahl K, Lagergren J. Lymphocytic esophagitis: a histologic subset of chronic esophagitis. Am J Clin Pathol 2006;125:432-437.

5 Cohen S, Saxena A, Waljee AK et al. Lymphocytic esophagitis: a diagnosis of increasing frequency. J Clin Gastroenterol 2012;46:828-832.

6 Haque S, Genta RM. Lymphocytic oesophagitis: clinicopathological aspects of an emerging condition. Gut 2012;61:1108-1114.

7 Schneider NI, Plieschnegger W, Geppert M et al. Validation study of the Esohisto consensus guidelines for the recognition of microscopic esophagitis (histoGERD Trial). Hum Pathol 2014;45:994-1002.

8 Ganz RA, Utley DS, Stern RA et al. Complete ablation of esophageal epithelium with a balloon-based bipolar electrode: a phased evaluation in the porcine and in the human esophagus. Gastrointest Endosc 2004;60: 1002-1010.

9 Halsey KD, Greenwald BD. Cryotherapy in the management of esophageal dysplasia and malignancy. Gastrointest Endosc Clin N Am 2010;20: 75-87 vi-vii.

10 Purdy JK, Appelman HD, Golembeski CP et al. Lymphocytic esophagitis: a chronic or recurring pattern of esophagitis resembling allergic contact dermatitis. Am J Clin Pathol 2008;130:508-513.

11 Loke SS, Yang KD, Chen KD et al. Erosive esophagitis associated with metabolic syndrome, impaired liver function, and dyslipidemia. World J Gastroenterol 2013;19:5883-5888.

12 Basseri B, Levy M, Wang HL et al. Redefining the role of lymphocytes in gastroesophageal reflux disease and eosinophilic esophagitis. Dis Esophagus 2010;23: 368-376.

13 Lind A, Koenderman L, Kusters JG et al. Squamous tissue lymphocytes in the esophagus of controls and patients with reflux esophagitis and Barrett's esophagus are characterized by a non-inflammatory phenotype. PLoS One 2014;9:e106261.

14 Demir D, Doganavsargil B, Sarsik B et al. Is it possible to diagnose infectious oesophagitis without seeing the 
causative organism? A histopathological study. Turk J Gastroenterol 2014;25:481-487.

15 Mangano MM, Antonioli DA, Schnitt SJ et al. Nature and significance of cells with irregular nuclear contours in esophageal mucosal biopsies. Mod Pathol 1992;5:191-196.

16 Cucchiara S, D'Armiento F, Alfieri E et al. Intraepithelial cells with irregular nuclear contours as a marker of esophagitis in children with gastroesophageal reflux disease. Dig Dis Sci 1995;40: 2305-2311.
17 Ebach DR, Vanderheyden AD, Ellison JM et al. Lymphocytic esophagitis: a possible manifestation of pediatric upper gastrointestinal Crohn's disease. Inflamm Bowel Dis 2011;17:45-49.

18 Basseri B, Vasiliauskas EA, Chan O et al. Evaluation of peripapillary lymphocytosis and lymphocytic esophagitis in adult inflammatory bowel disease. Gastroenterol Hepatol (N Y) 2013;9:505-511.

19 Chandan VS, Murray JA, Abraham SC. Esophageal lichen planus. Arch Pathol Lab Med 2008;132: 1026-1029. 